\title{
Supporting Information for: Ensemble Inhomogeneity of Dielectric Functions in Cs-doped Tungsten Oxide Nanoparticles
}

\author{
Keisuke Machida and Kenji Adachi* \\ Ichikawa Research Center, Sumitomo Metal Mining Co., Ltd., Ichikawa, Chiba 272-8588 Japan \\ E-mail:kenji_adachi@ni.smm.co.jp
}

\section{Effect of dipole-dipole interactions on the absorption bandwidth}

For assessing the effect of dipole-dipole interactions on the near-infrared absorption profiles of $\mathrm{Cs}_{0.33} \mathrm{WO}_{3}$ (CWO) dispersions, three kinds of CWO dispersions with different inter-dipolar distance have been prepared. Two among three are liquid dispersions in methyl isobutyl ketone (MIBK) solvent with CWO concentrations 0.005 wt. $\%$ and 0.05 wt.\%. They are prepared by diluting a $20 \mathrm{wt} . \%$-dispersion liquid manufactured by bead-milling to the average particle size of $20 \mathrm{~nm}$. For the $0.005 \mathrm{wt} . \%$-dispersion, one CWO particle is contained in the liquid volume of $7.50 \times 10^{8} \mathrm{~nm}^{3}$, calculated with the density of particles and solvent as $7.16 \mathrm{~g} / \mathrm{cm}^{3}$ and $0.80 \mathrm{~g} / \mathrm{cm}^{3}$, respectively. This leads to the mean inter-particle distance as $908 \mathrm{~nm}$. Similarly it is calculated as $440 \mathrm{~nm}$ for the 0.05 wt.\%-dispersion.

Acrylic resin coating containing 27 wt. \% CWO nanoparticles (NPs) has been made on a PET film. UV curable resin, ARONIX UV-3701 (Toagosei Co., Ltd., Tokyo, Japan), and 20 wt.\% CWO dispersion liquid were mixed at

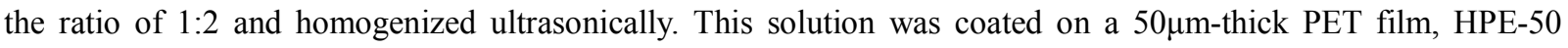
(Teijin DuPont Films Japan Limited, Tokyo, Japan), with a No.10 wire bar. After $70{ }^{\circ} \mathrm{C}$ drying for 1 minute, UV light was illuminated until the resin has been cured. The coating layer holds one NP per $7.18 \times 10^{4} \mathrm{~nm}^{3}$ with the resin density of $1.20 \mathrm{~g} / \mathrm{cm}^{3}$, and the average inter-dipolar distance is calculated as $41.6 \mathrm{~nm}$.

Absorbance of each dispersion ink and film was measured with a spectrophotometer and shown normalized in Fig. S1. Baselines were set using blank solvent and blank acrylic resin, respectively. Clearly, every dispersion shows almost the same absorption profile, proving that the dipolar interaction does not affect the absorption bandwidth of CWO NPs. Minor variance in the curvilinear feature can be explained by differences in the refractive index of surrounding media and/or the degree of pulverization.

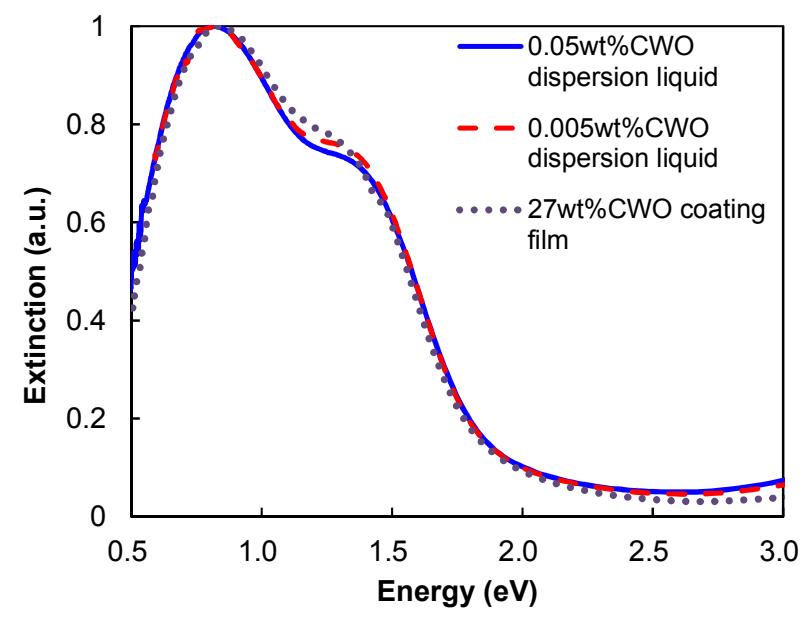

Fig. S1 Extinction cross sections measured for CWO dispersions of different inter-dipolar distances.

\footnotetext{
* Author to whom correspondence should be addressed.
} 


\section{TEM cross sectional image of acid-etched CWO plate}

Sintered CWO plate was heavily etched with hydrochloric acid to urge Cs desorption from the surface. Specimen surface was monitored by optical reflectance successively on repeated etching, which was finished when appreciable change appeared in reflectance. Thus, the overall acid-etching process consisted of $1 \% \mathrm{HCl}$ for 14 hours at $70{ }^{\circ} \mathrm{C}, 6 \% \mathrm{HCl}$ for 7 hours at $70{ }^{\circ} \mathrm{C}, 35 \% \mathrm{HCl}$ for 24 hours at $90{ }^{\circ} \mathrm{C}$ and $35 \% \mathrm{HCl}$ for 240 hours at room temperature. The etched surface was rinsed with ion-exchanged water and dried in air.

TEM cross sectional image near the surface of the heavily-etched CWO plate is shown in Fig. S2. The specimen is polycrystalline with grain boundaries observed. Three points, $d_{1}, d_{2}$, and $d_{3}$ correspond to surface, grain interior and grain boundary, respectively, where electron diffraction patterns were taken. Electron diffraction patterns from $d_{1}$ and $d_{3}$ suggests inclusion of amorphous phase.

EDX analysis was made and the result is shown in the table. Cs/W at both surface and grain boundary is around 0.07 , considerably less than the value at grain interior. It indicates that the Cs atoms are extracted from surface and along grain boundaries.

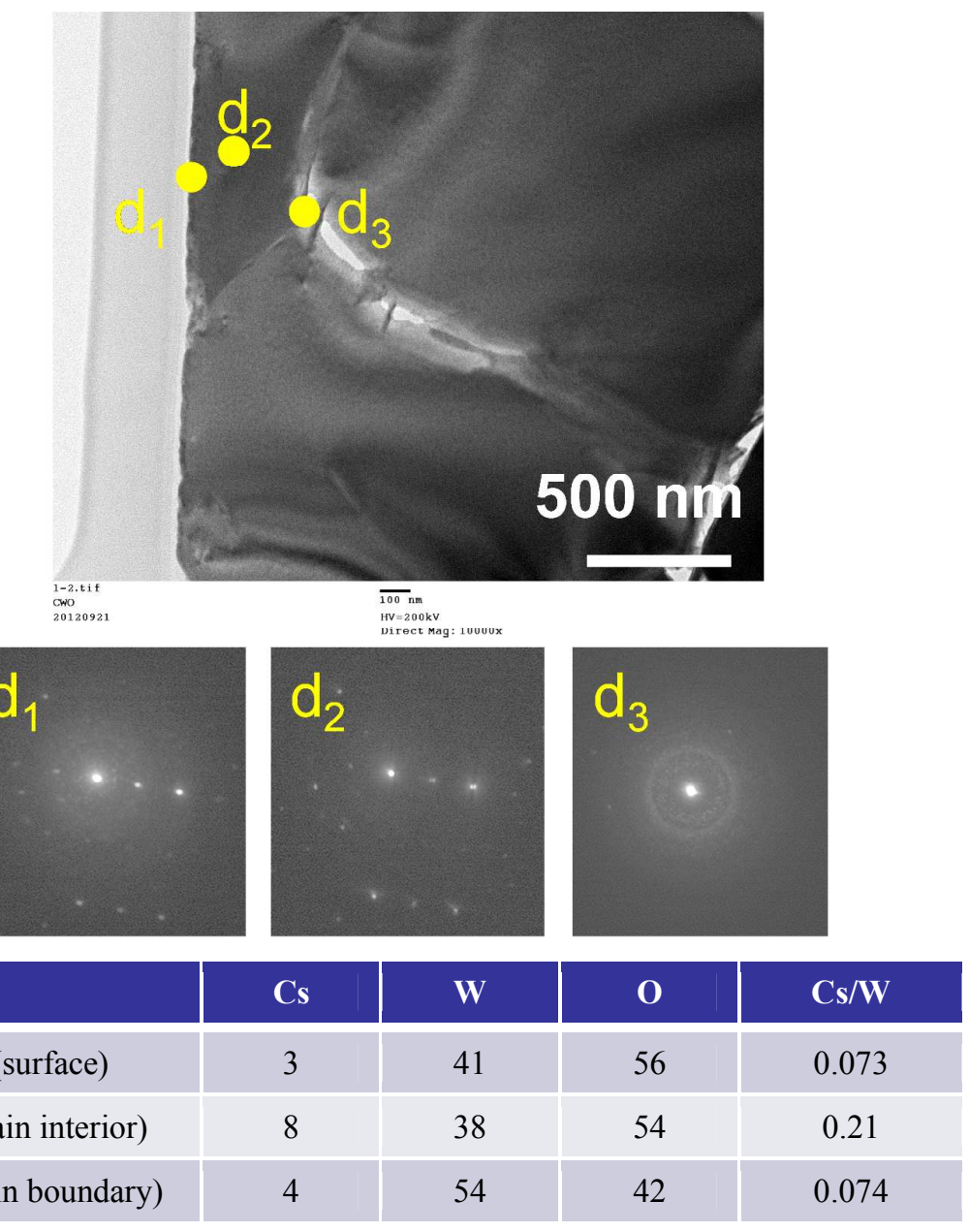

Fig. S2 TEM cross sectional image of CWO plate heavily-etched with hydrochloric acid, with electron diffraction patterns and EDX compositions (in at.\%) taken from the surface, grain interior, and grain boundary, respectively. 


\section{Tauc Plot for CWO}

Frequency-dependent absorption coefficient $\alpha(\omega)$ of bulk CWO was obtained from $\alpha=4 \pi k / \lambda$, where $k(\omega)$ is an imaginary part of refractive index measured with ellipsometry, $\lambda=c / \omega$ is wavelength and $c$ is the speed of light. Tauc plot is drawn in Fig. S3, indicating the direct band gap to be $3.05 \mathrm{eV}$.

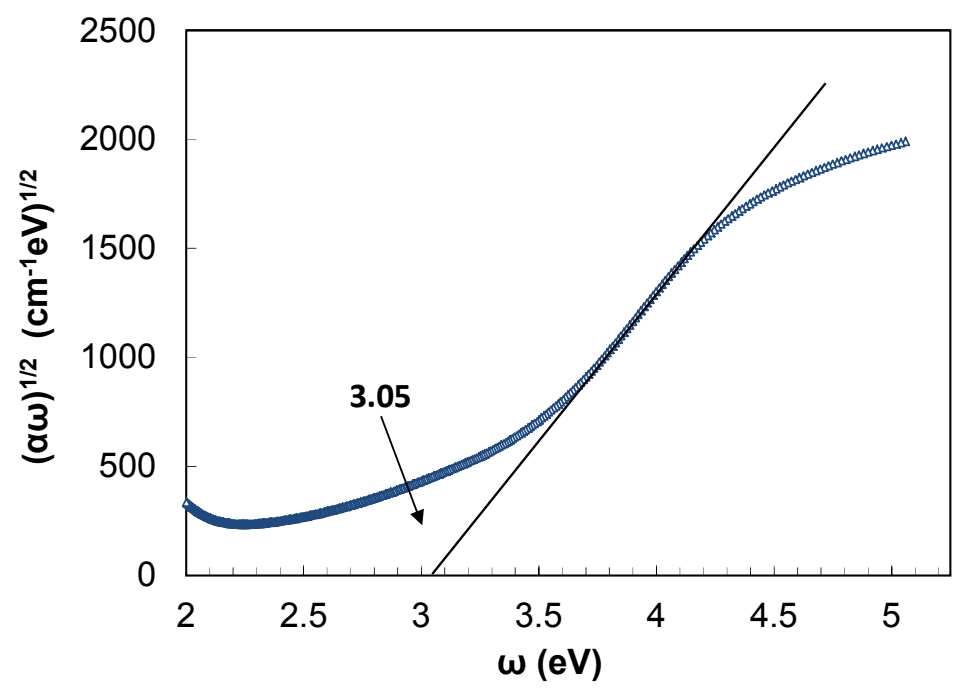

Fig. S3 Tauc plot for band gap in $\mathrm{Cs}_{0.33} \mathrm{WO}_{3}$. 


\section{The effect of integrating number of particles in NP ensemble}

In order to determine the minimum number of NPs to describe behaviors of the ensemble consisting of the molar-order number of NPs, Mie-scattering integration calculations as described in the text have been made for 10 , 100, 1,000, 10,000 and 100,000 NPs for integration. Best-fitted mean and standard deviation values shown in Table 1 in the text were employed for the calculations.

As shown in Fig. S4, the calculated extinctions have changed gradually for small $\mathrm{n}$, depending on the random number sequences used. Curves are saturated to the final form with more than 10,000 elements. Therefore, we have taken 100,000 as the number for integration in the analysis.

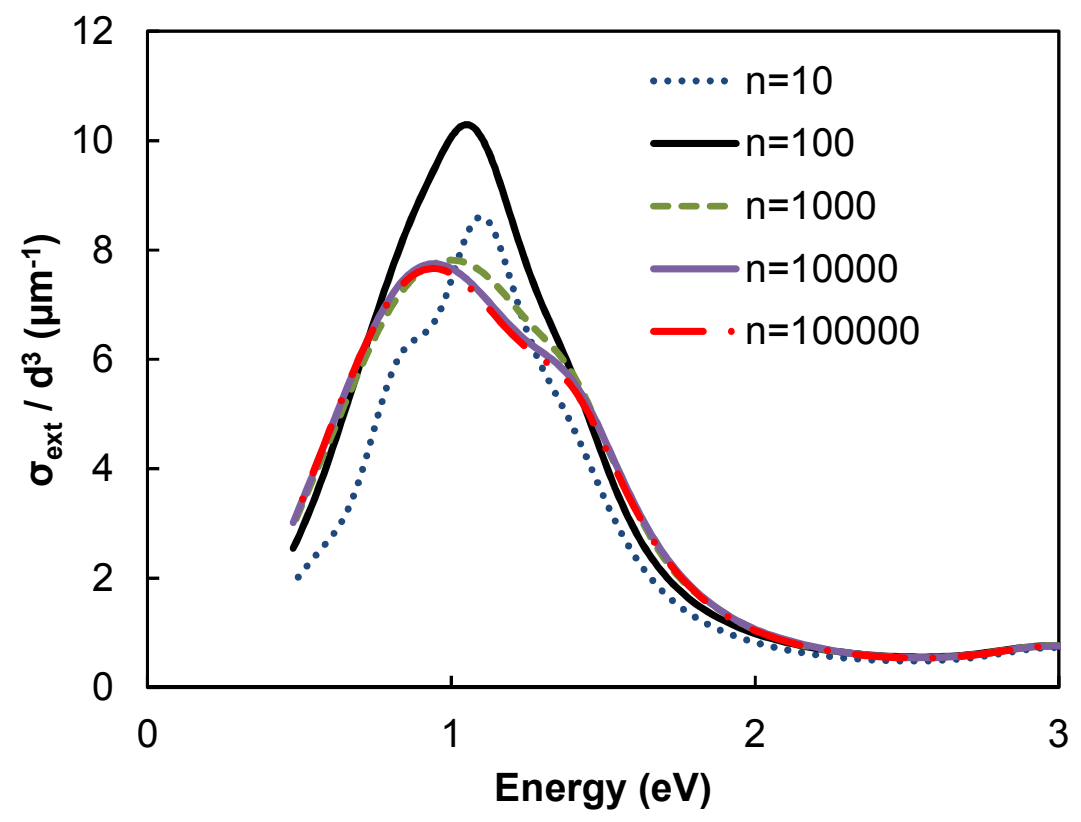

Fig. S4 Effect of numbers of particles on integrated $\sigma_{\text {ext }}$. 